\title{
Real Time Observation of the Echo Source of Systolic Hump in Hypertrophic Obstructive Cardiomyopathy with High Speed Ultrasono-Cardiotomography
}

\author{
Tadashi Kambe, M.D., Norio Hrbi, M.D., Kinya Nishimura, M.D., \\ Toshinori Sakakibara, M.D., Tadayuki Kato, M.D., \\ Takemi Arakawa, M.D., Yoichi Fukui, M.D., \\ Arata Miwa, M.D., Hisao Tada, M.D., Nobuo Sakamoto, M.D., \\ and Kohzo Hisanaga, M.D., B.S.*
}

\section{SUmmary}

The present study was undertaken to discuss the echo source of systolic hump in hypertrophic obstructive cardiomyopathy (HOCM) from a viewpoint of B-mode echocardiography. Cross-sectional images were obtained from 4 patients with HOCM using a Sonolayergraph of Toshiba, SSL-51H. This equipment is characterized by its high speed mechanical sector scanning and wider angle.

Recordings were made with ordinary $35 \mathrm{~mm}$ camera or Polaroid in conjunction with $8 \mathrm{~mm}$ cinecamera.

Our data showed that systolic hump in this lesion was not caused by anterior systolic movement of anterior mitral leaflet, but was emanated from the chordae tendineae attached to the anterior or posterior mitral leaflet. In systole, the anterior mitral leaflet moved backward for closure, while the chordae tendineae approached the bulged interventricular septum resulting in the formation of systolic hump.

\section{Additional Indexing Words :}

Systolic anterior motion Idiopathic hypertrophic subaortic stenosis B-mode echocardiography M-mode echocardiography Left heart catheterization

CTANDARD M-mode echocardiography is an indispensable technique for $\mathcal{N}$ the evaluation of hypertrophic obstructive cardiomyopathy. However, it is not suitable for the spacial presentation of intracardiac structure in detail, since it is essentially one-dimensional.

From the Third Department of Internal Medicine, Nagoya University, School of Medicine, Tsurumai-cho 65, Showa-ku, Nagoya 466 and Mitsubishi Nagoya Hospital,* Sotodoi-cho 48, Atsutaku, Nagoya 456.

Address for reprints: Dr. Tadashi Kambe, Third Department of Internal Medicine, Nagoya University, School of Medicine, Tsurumai-cho 65, Showa-ku, Nagoya 466, Japan.

Received for publication March 16, 1977. 
Since the advent of cross-sectional echocardiography, there have been a variety of documents ${ }^{1)-7)}$ regarding hypertrophic obstructive cardiomyopathy. However, attention has been paid mostly to the mechanism of left ventricular obstruction and the echo source of systolic hump still remains to be solved.

A recent report presented by Martin and associates $^{8)}$ has given rise to further study on the systolic hump in this lesion.

The purpose of the present paper is to discuss the echo source of systolic hump or SAM (systolic anterior motion) in hypertrophic obstructive cardiomyopathy by real time observation with high speed ultrasono-cardiotomography.

\section{Materials and Methods}

Four patients, 2 males and 2 females, ranging in age from 16 to 44 years were studied. The diagnosis was established in each case by catheterization and angiocardiography in conjunction with standard M-mode echocardiography. Findings are listed in Table I. All of the subjects had a prominent systolic hump in M-mode echocardiograms.

Table I. Case Materials

\begin{tabular}{c|c|c|c|c|c|c}
\hline \multirow{2}{*}{ No. } & Name & Age & Sex & $\begin{array}{c}\text { SAM in } \\
\text { M-mode } \\
\text { UCG }\end{array}$ & \multicolumn{2}{|c}{ Systolic LV Pressure (mmHg) } \\
\hline 1 & K. G. & 22 & M & yes & 140 & outflow \\
2 & Y. S. & 24 & F & yes & 115 \\
3 & K. O. & 16 & M & yes & 170 & 130 \\
4 & Y.S. & 44 & F & yes & 290 & 125 \\
\end{tabular}

$\mathrm{SAM}=$ systolic anterior motion or systolic hump, LV=left ventricle

Ultrasound recordings of the systolic hump were made using a Sonolayergraph of Toshiba, Model SSL-51H and a $3 \mathrm{MHz}$ transducer. As previously reported from our laboratory, ${ }^{9)}$ this equipment is characterized by its high speed mechanical sector scanning and real time observation of intracardiac structures.

All the subjects were examined in the supine position. For better acoustic coupling between chest surface and transducer, the so-called "proximity immersed method "9) was used, in which the probe was soaked in a vinyl bag filled with castor oil. The distance from the chest surface was approximately $5 \mathrm{~mm}$. A crosssection along the left ventricular long axis was usually employed.

Two methods ${ }^{9}$ were utilized to record cross-sectional images on the oscilloscopic screen. First, the photographs were taken by an ordinary $35 \mathrm{~mm}$ camera or Polaroid in synchrony with ECG, and occasionally taken at a shutter speed of $1 / 10$ or $1 / 15 \mathrm{sec}$ without a triggering circuit. The second approach was a cinematographic method with a commercially available $8 \mathrm{~mm}$ cinecamera. 


\section{Results}

A representative M-mode echocardiogram is shown in Fig. 1. This picture was taken from a 16-year-old male with hypertrophic obstructive cardiomyopathy (K.O.). As indicated by a white arrow, a prominent systolic hump or SAM is visualized in systole. The interventricular septum (IVS) is extremely thickened and encroaches upon the left ventricular outflow tract. The systolic pressure gradient between left ventricular outflow and apex was $45 \mathrm{mmHg}$ according to the hemodynamic finding (Table I).

In order to detect the echo source of the systolic hump in this patient, two-dimensional echocardiograms were obtained along the left ventricular long axis as shown in Fig. 2. In diastole (left picture), the anterior mitral leaflet (AML) approaches the extremely thickened interventricular septum (IVS), while in systole (right picutre), the chordae tendineae (CT) are anter-

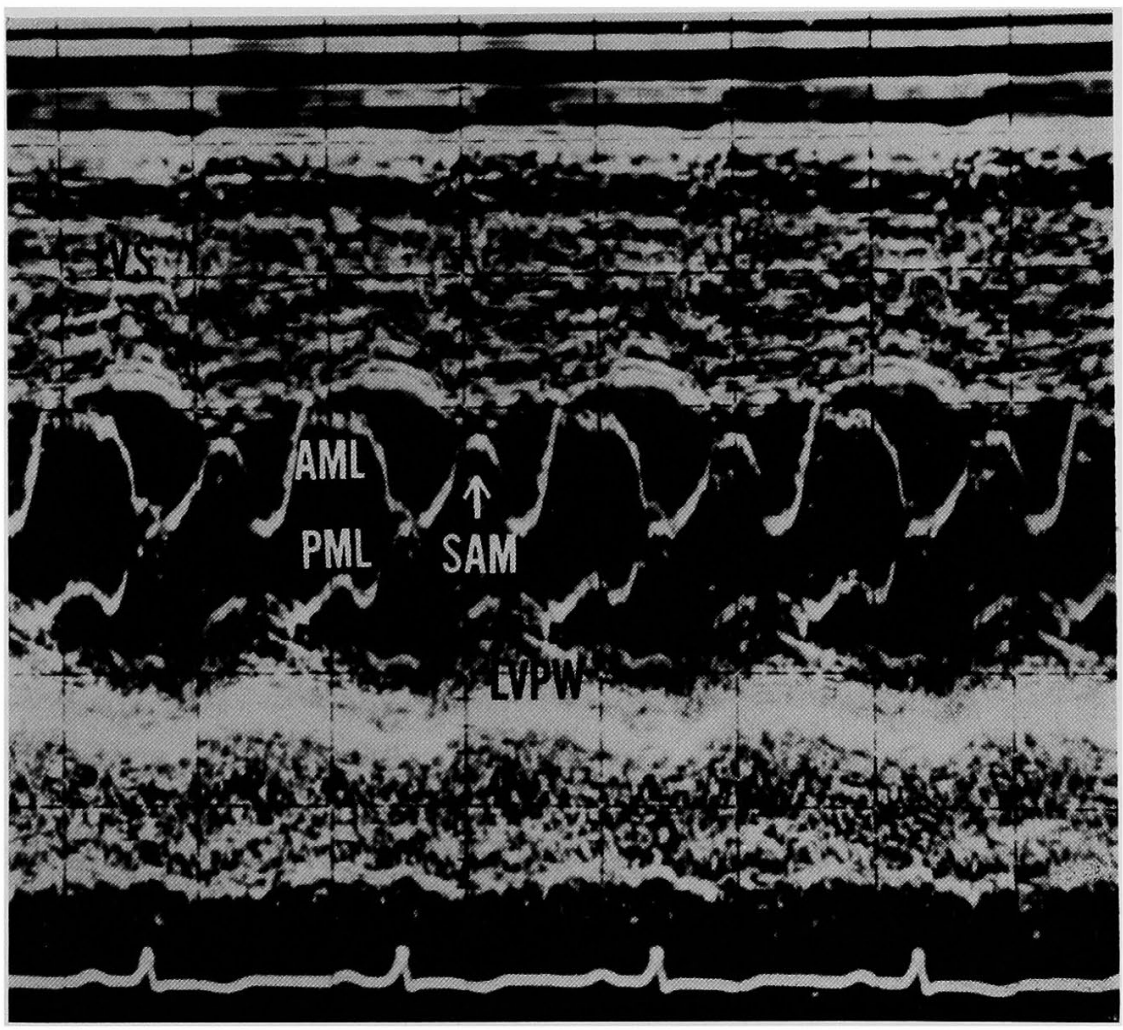

Fig. 1. A representative M-mode echocardiogram taken from a 16-yearold male with HOGM. As indicated by a white arrow, a prominent systolic hump is visualized in systole. IVS = interventricular septum, SAM=systolic hump or systolic anterior motion, $\mathrm{AML}=$ anterior mitral leaflet, $\mathrm{PML}=$ posterior mitral leaflet, LVPW= left ventricular posterior wall. 

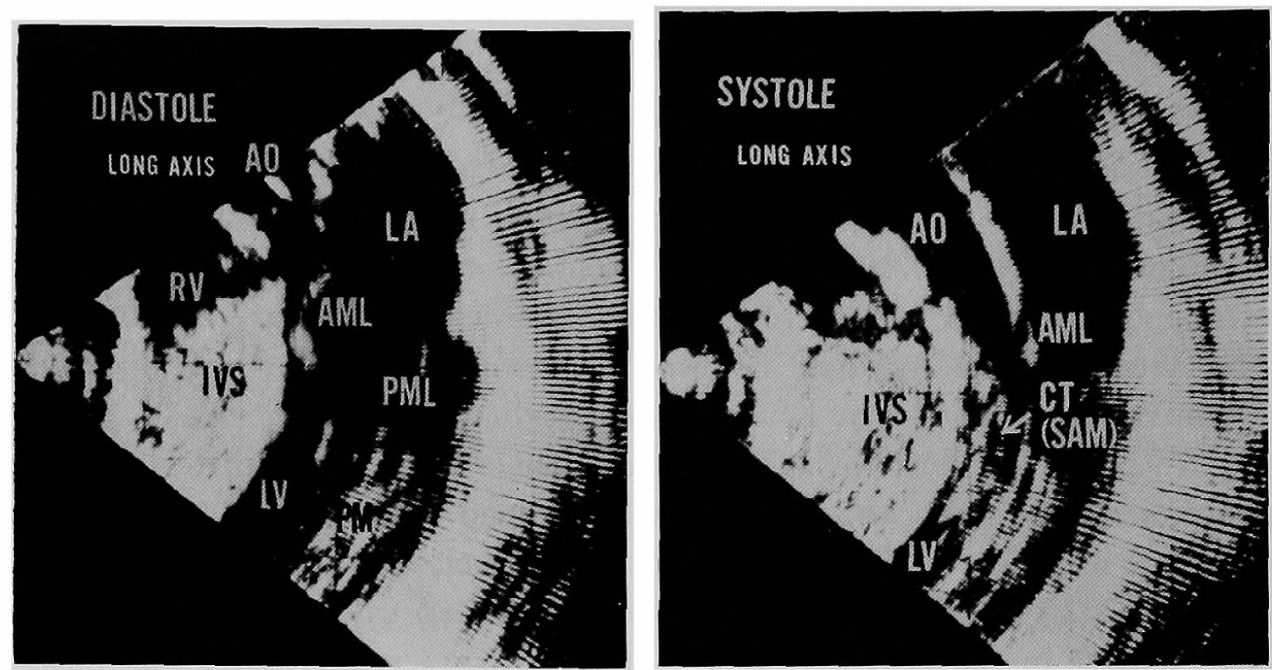

Fig. 2. Cross-sectional echocardiograms from the same patient as in Fig. 1. These images were obtained along the long axis of left ventricle. In diastole (left picture), the AMI, fully opens and approaches the extremely thickened IVS, while in systole (right picture), the chordae tendineae (white arrow) are anteriorly pulled toward the outflow tract of left ventricle. At this moment, the AML retreats backward in the opposite direction. RV= right ventricle, $A O=$ aorta, $L A=$ left atrium, $L V=$ left ventricle, $P M=$ papillary muscles, CT $=$ chordae tendineae, CT $(\mathrm{SAM})=$ the chordae tendineae are the echo source of systolic hump.

iorly pulled toward the outflow tract of the left ventricle. At this moment, the anterior mitral leaflet moves backward in the opposite direction. Furthermore, based upon a real time observation by $8 \mathrm{~mm}$ cinematography, it is impossible for the mitral leaflet to be an echo source of systolic hump, since it is located well away from the outflow tract of left ventricle when the chordae tendineae maximally approach the outflow.

Fig. 3 shows another example of SAM in M-mode echocardiogram taken of a 22-year-old male (K.G.). A white arrow indicates a prominent systolic hump. As shown in Table I, there was a systolic pressure gradient of 25 $\mathrm{mmHg}$ in the left ventricular cavity.

B-mode echocardiograms of the same patient as in Fig. 3 are demonstrated in Fig. 4. The cross-sectional images were obtained through the long axis of left ventricle. Similar to the K.O.'s case, the chordae tendineae comes into close contact with the interventricular septum in systole (left picture), in contrast with the anterior mitral leaflet going back for closure. In addition, the anterior mitral leaflet moves forward maximally in diastole for opening (right picture). This phenomenon implies that the SAM is generated by the chordae tendineae. 


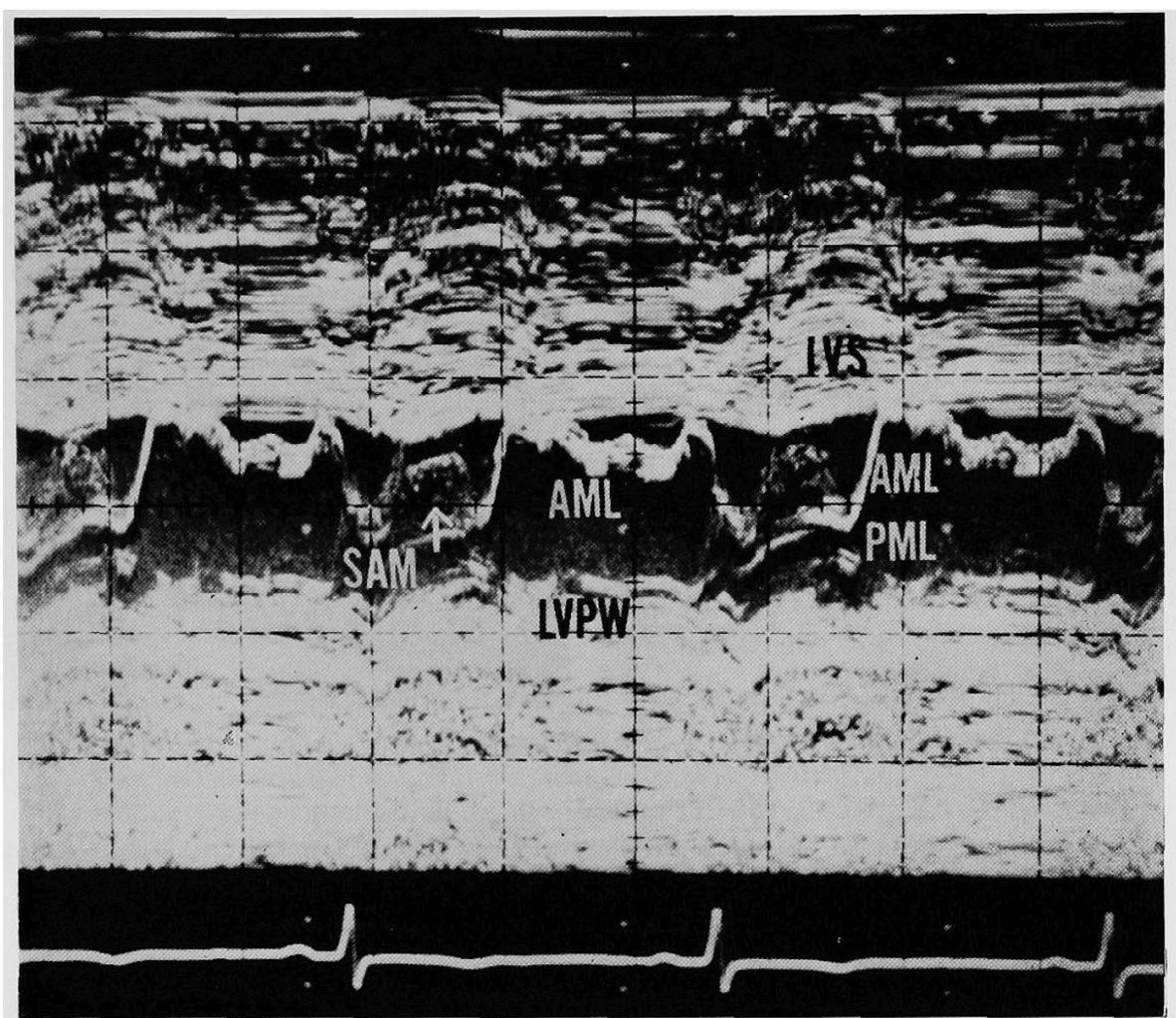

Fig. 3. M-mode echocardiogram from a 22-year-old male with HOCM. A white arrow shows a prominent SAM in systole. The AML fully opens in diastole and comes in touch with the thickened IVS.

The interventricular septum is extremely thickened and encroaches upon the left ventricular cavity in concert with the hypertrophied papillary muscles (PM).

The summary of results is listed in Table II; in all the subjects, the systolic hump is generated by the chordae tendineae attached to the anterior or posterior mitral leaflet.

\section{Discussion}

Conventional M-mode echocardiography has been successfully used in the diagnosis of idiopathic hypertrophic subaortic stenosis. And the systolic hump is considered to be one of the most important diagnostic criteria of this lesion. There have been many reports ${ }^{1)-7)}$ regarding the systolic hump as derived from abnormal forward movement of anterior mitral leaflet during systole. 

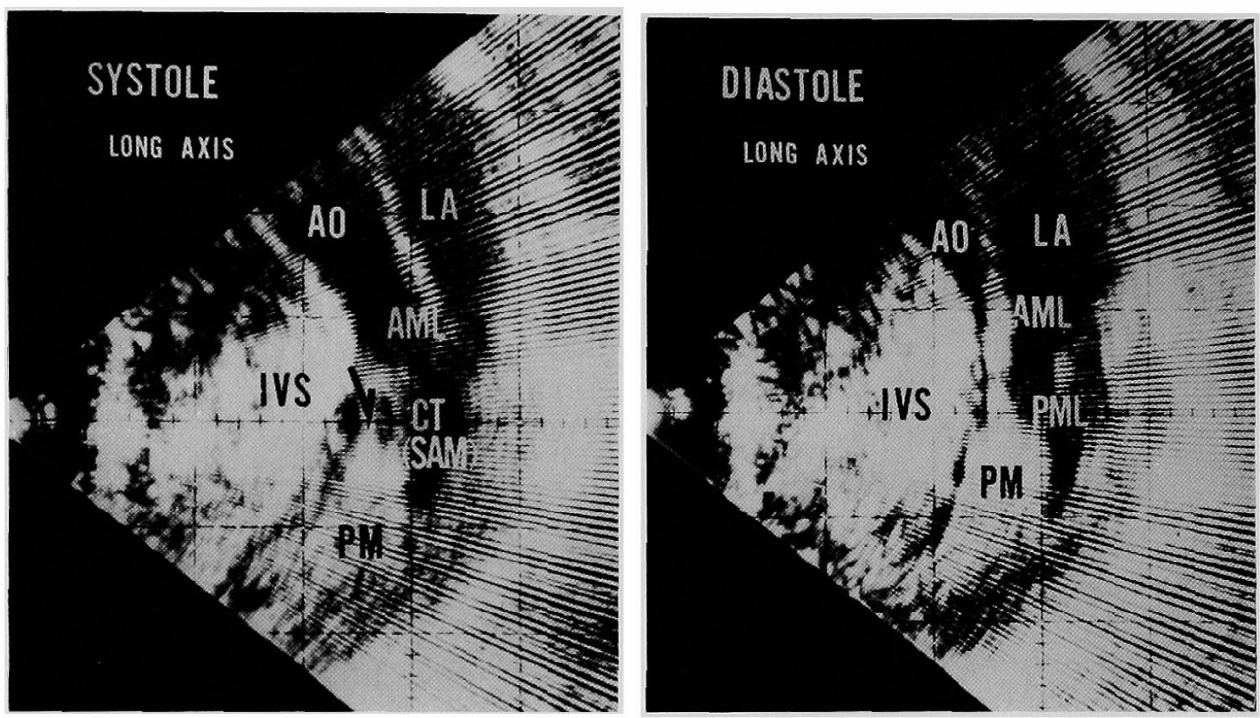

Fig. 4. Cross-sectional echocardiograms from the same patient as shown in Fig. 3. These pictures were taken along the long axis of left ventricle. In systole (left panel), the chordae tendineae (black arrow) are anteriorly pulled and closely contact the thickened IVS, whereas the AML moves backward. In diastole (right panel), the AML approaches the IVS. The systolic hump is generated by the chordae tendineae with certainty.

Table II. Echo Source of SAM

\begin{tabular}{c|c|c|c|c|c}
\hline No. & Name & Age & Sex & $\begin{array}{c}\text { SAM in } \\
\text { M-mode UCG }\end{array}$ & Echo Source of SAM \\
\hline 1 & K. G. & 22 & M & yes & chordae tendineae \\
2 & Y.S. & 24 & F & yes & chordae tendineae \\
3 & K. O. & 16 & M & yes & chordae tendineae \\
4 & Y.S. & 44 & F & yes & chordae tendineae
\end{tabular}

In this study we attempted to protest against this theory since there is a great discrepancy between our observation and those in the documents ${ }^{1-5}$ ) previously reported by other authors. The analysis of the systolic hump or SAM in their studies were mostly based on M-mode echocardiography. It lacks the spacial orientation of mitral apparatus since it is essentially onedimensional.

In this connection, B-mode echocardiography is thought more useful for two-dimensional display of fine intracardiac structures. Henry and associates $^{6}$ commented on the mechanism of left ventricular outflow tract obstruction in this lesion using cross-sectional echocardiography. However, chordal contribution to the SAM formation was not mentioned in their report. Furthermore, Cohen and associates ${ }^{7)}$ noted with B-mode echocardiography that sys- 
tolic hump may present the motion of only a small part of the mitral leaflet, perhaps the free edge or redundant valve tissue.

In order to identify the echo source of systolic hump, it is necessary to carefully observe the subtle movements of mitral apparatus and to distinguish mitral leaflet from chordae tendineae using motion picture or video system. And it is also important to repeat the real time observation of chordal area.

According to our experience, anterior mitral leaflet maximally approaches the bulged interventricular septum in diastole, whereas it is positioned well away from the septum in systole. In contrast, the chordae tendineae show a rapid forward movement toward the thickened interventricular septum in systole, resulting in the formation of systolic hump.

It is most likely that systolic hump is not generated by abnormal systolic motion of anterior mitral leaflet, but derived from the chordae tendineae attached to anterior or posterior mitral leaflet. It is impossible that the anterior mitral leaflet maximally contacts the interventricular septum in midsystole, because it is located well away from the septum for its closure.

The fallacy committed by other author( ${ }^{1)-5}$ is probably caused by the difficult spacial orientation of echo source with M-mode echocardiography. At present, however, it is not always easy in each case to determine which chordal system, anterior or posterior, plays a main role in the genesis of systolic hump.

It is not clear that chordae tendineae contribute to the left ventricular obstruction, but a combination of the septum and papillary muscles is thought to be a major factor causing the obstruction. The chordae tendineae may be sucked by a Venturi's effect in contrast with the anterior mitral leaflet retreating backward.

Recently, Nimura and associates ${ }^{10)}$ protested against the theory that systolic hump is caused by anterior systolic motion of mitral leaflet. We insist here on the importance of real time observation of heart with B-mode echocardiography, since it provides a spacial orientation of echo source of systolic hump.

\section{ACKNOWLEDGEMENTS}

We thank Drs. Noboru Yamazaki and Jun Akunc for their cooperation.

\section{REFERENCES}

1. Shah PM, Gramiak R, Kramer DH: Ultrasound location of left ventricular outflow tract obstruction in hypertrophic obstructive cardiomyopathy. Circulation 40:3, 1969

2. Popp RL, Harrison DC: Ultrasound in the diagnosis and evaluation of therapy of idiopathic 
hypertrophic subaortic stenosis. Circulation 40: 905, 1969

3. Shah PM, Gramiak R, Adelman AG, Wigle ED: Role of echocardiography in diagnostic and hemodynamic assessment of hypertrophic subaortic stenosis. Circulation 45: 516, 1972

4. Feigenbaum H: Clinical applications of echocardiography. Prog Cardiovasc Dis 14: 531, 1972

5. Henry WI., Clark CE, Epstein SE: Echocardiographic identification of the pathognomonic anatomic abnormality of IHSS. Circulation 47:225, 1973

6. Henry WL, Clark CE, Griffith JM, Epstein SE: Mechanism of left ventricular outflow tract obstruction in patients with obstructive asymmetric septal hypertrophy (idiopathic hypertrophic subaortic stenosis). Am J Cardiol 35: 337, 1975

7. Cohen MV, Teichholtz LE, Gorlin R: B-scan ultrasonography in idiopathic hypertrophic subaortic stenosis. Study of left ventricular outflow tract and mechanism of obstruction. Brit Heart J 38: 595, 1976

8. Martin RP, French JW, Pettman MM, Popp RL: Analysis of hypertrophic subaortic stenosis by wide angle two-dimensional phased array echocardiography. Presented at the First Meeting of the World Federation for Ultrasound in Medicine and Biology, San Francisco, August $3-7,1976$

9. Nishimura $K$, Hibi $N$, Fukui $Y$, Arakawa $T$, Tatematsu $H$, Miwa A, Tada $H$, Kambe T, Nakagawa K, Takemura $\mathrm{X}$ : Real time observation of cardiac movement and structures in congenital and acquired heart diseases employing high-speed ultrasononcardiotomography. Am Heart J 92: 340, 1976

10. Nimura Y, Sakakibara H, Matsuo H, Matsumoto M, Miyatake K, Nagata S, Beppu S, Tamai $\mathbf{M}$, Senda $\mathbf{K}$, Abe $\mathrm{H}$ : Studies on left ventricular anatomy and events in hypertrophic cardiomyopathy with ultrasono-cardiotomography in special reference to obstructive signs. Shinzo 7: 1461, 1975 (in Japanese) 